\title{
Optic disc neovascularisation in diabetic retinopathy: I. A system for grading proliferation at the optic nerve head in patients with proliferative diabetic retinopathy
}

\author{
YUVAL YASSUR, ${ }^{1}$ LINDA W. PICKLE, ${ }^{2}$ STUART L. FINE, \\ LAWRENCE SINGERMAN, DAVID H. ORTH, AND ARNALL PATZ
}

From the Retinal Vascular Service, Wilmer Institute, Johns Hopkins University and Hospital, Baltimore, USA

SUMMARY An anatomical taxonomy for neovascular proliferation of the optic nerve head in patients with proliferative diabetic retinopathy is presented. The technique consists in comparing (A) number of involved quadrants, (B) calibre of vessels, (C) neovascular density, (D) neovascular area, (E) plane of proliferation, and (F) fluorescein angiographic findings. A similar taxonomy is used to describe fibrous tissue proliferation at the disc. With these criteria a series of standard photographs have been utilised in a numerical grading system. This system has proved useful in documenting the natural history and response to photocoagulation therapy for diabetic disc neovascularisation.

Diabetic retinopathy is often associated with a neovascular proliferation at the optic nerve head. This growth may be primarily vascular or fibrous or contain significant elements of both simultaneously. Previous methods of classifying these changes were incorporated in the Airlie House System $^{1}$ as well as others. ${ }^{2-7}$ Our more detailed classification of disc neovascularisation has facilitated its more precise documentation.

\section{Material and Methods}

All the patients with proliferative diabetic retinopathy seen in our laboratory had colour stereoscopic photographs taken of the optic disc. A Zeiss fundus camera and Kodachrome 25 colour film were used. Fluorescein angiography of the optic disc was also performed on each eye.

Disc proliferation was defined as any vascular or fibrous tissue emanating from the optic nerve head or located within 1 disc diameter of the disc margin. The vascular and fibrous elements were evaluated separately. Arbitrary criteria for a scale of relative importance were established. In this manner a scoring method was created to grade the prolifera-

${ }^{1}$ Current address for reprints Beilinson Hospital and Medical Center, Petach-Tikva, Israel.

${ }^{2}$ From the Department of Biostatistics, Johns Hopkins University and Hospital. tion in regard to the separate neovascular and fibrous changes.

The disc neovascularisation (NVD) was reported according to the following parameters:

A. The number of quadrants (1-4) of the optic disc in which neovascularisation could be identified (Figs. 1 $a-d$ ).

B. The calibre of the new blood vessels. It was called stage I when the largest diameter of the new vessels was less than half the size of the first branch of the central retinal artery; stage II when the new vessel calibre was between the half size and less than full-size of that branch artery; and stage III when the new vessel's largest diameter was equal to or greater than that branch artery (Figs. 2a-c).

C. Neovascular density was evaluated in comparison to a series of standard photographs. Four standards were established as shown in Figs. 3a-d. The stage of density was determined according to the standard photograph it resembled most (stages I-IV).

D. Area of neovascularisation was determined by measuring disc diameters of NVD on the stereoscopic photographs. It was called stage I when the total area was up to $2 \mathrm{DD}$; stage II when it was up to $5 \mathrm{DD}$; stage III when it was up to $10 \mathrm{DD}$; and stage IV when the total area was more than $10 \mathrm{DD}$.

E. The plane of proliferation of the new vessels was also determined by examining the stereoscopic photographs. It was called stage I when there was 

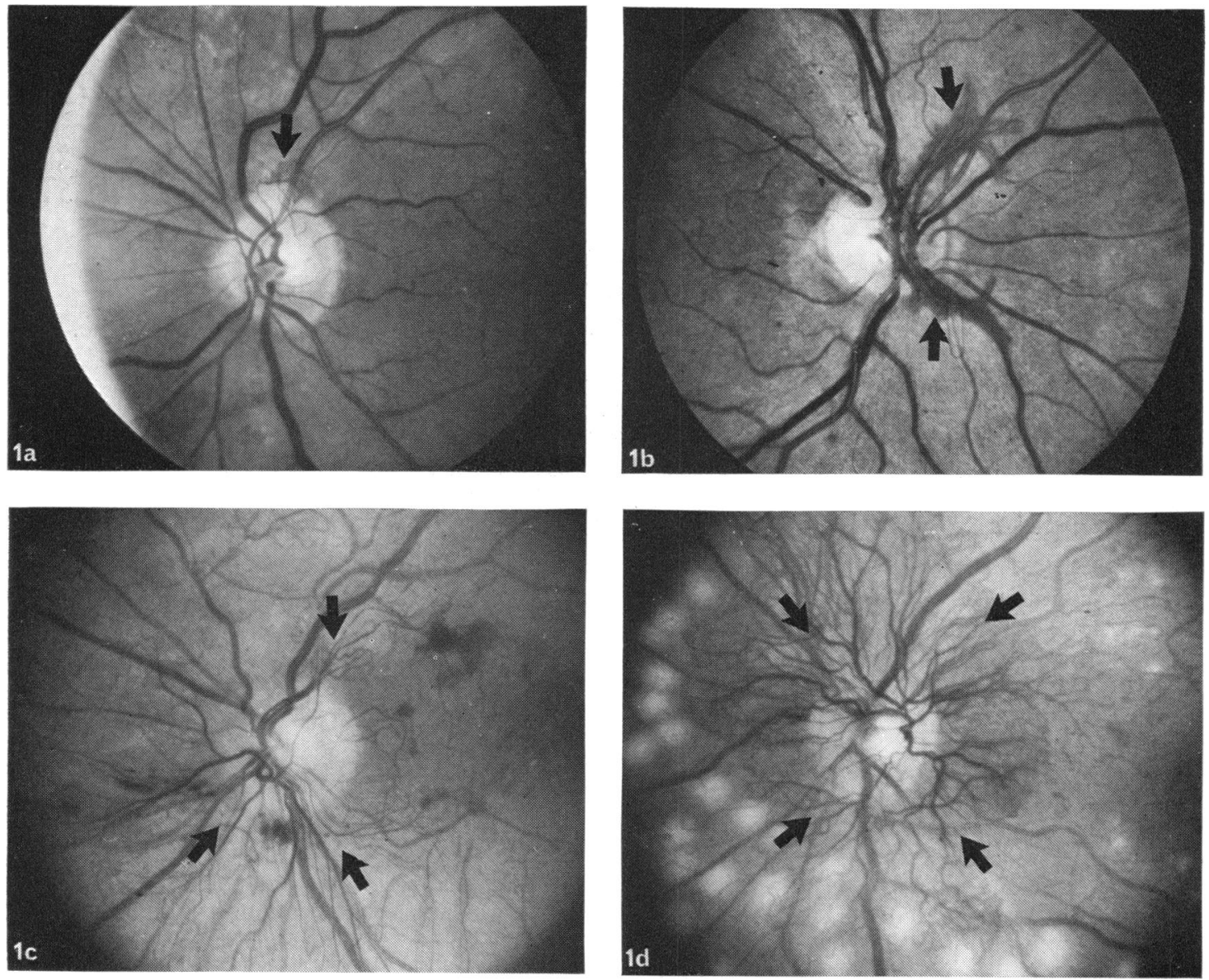

Figs. 1a-d Illustrations to show number of quadrants involved with neovascularisation: 1a. Arrows point to neovascularisation in 1 quadrant. 1b. Neovascularisation involves 2 quadrants of the disc. 1c. Disc neovascularisation involves 3 quadrants of the disc. 1d. Neovascularisation involves all quadrants of the disc.

less than one-quarter disc diameter of elevation; stage II when the elevation was between onequarter and 1 disc diameter; and stage III when the elevation was more than 1 disc diameter.

Fluorescein angiography was performed to demonstrate neovascular leakage. The dye leakage was recorded between 1 and 2 minutes after the injection, and was evaluated as stage 0 , I, II, or III, depending on the degree of dye leakage; a series of the following standard angiographic photographs were used for reference (Figs. $4 a-c$ ).

The parameters examined were quite dissimilar, and the stage number associated with each parameter could not be used to interrelate the findings, since they measured different criteria. For this reason the above listed parameters $\mathrm{A}-\mathrm{F}$ were given certain scores according to our previous observa- tions since 1960 on the natural history of approximately 600 patients with NVD that were not treated. We had concluded that the most important clinical parameters were the plane of proliferation and the total area of proliferation (parameters $D$ and $E$ in our list), since these were the key findings associated with vitreous haemorrhage, fibrosis, and traction retinal detachment. Therefore each of these parameters received a score of 40 points toward the total score of 100. The other four parameters (A, B, C, F) received a score of 5 points each. Each stage within each of the A-F parameters received their points as follows:

\section{Total no. of points for parameter \\ No. of stages composing the parameter}

and the points were added to each other when 

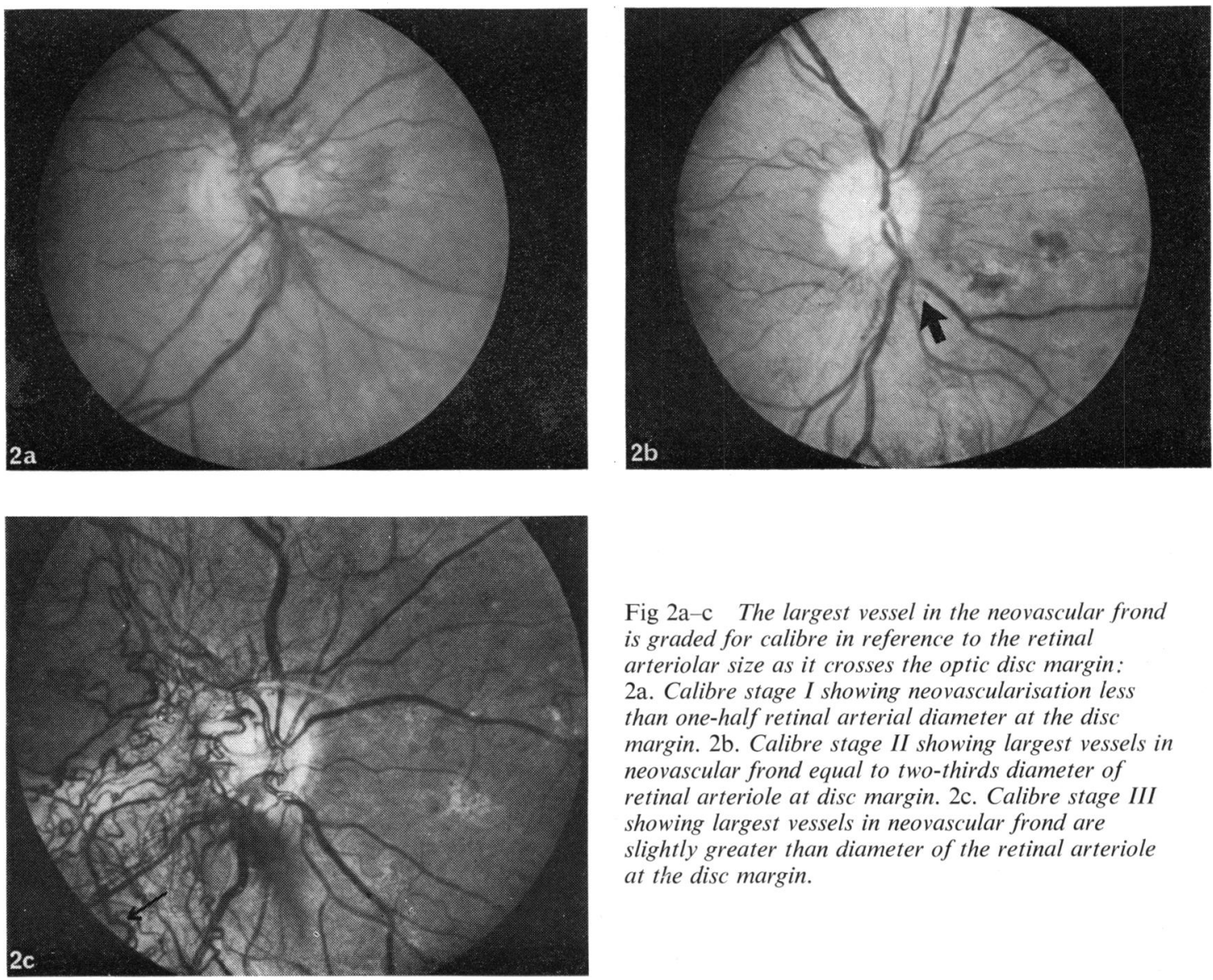

Fig $2 \mathrm{a}-\mathrm{c}$ The largest vessel in the neovascular frond is graded for calibre in reference to the retinal arteriolar size as it crosses the optic disc margin: 2a. Calibre stage I showing neovascularisation less than one-half retinal arterial diameter at the disc margin. 2b. Calibre stage II showing largest vessels in neovascular frond equal to two-thirds diameter of retinal arteriole at disc margin. 2c. Calibre stage III showing largest vessels in neovascular frond are slightly greater than diameter of the retinal arteriole at the disc margin.

moving up to the next stages of each parameter:

Table 1 Point system of stages and parameters of NVD

\begin{tabular}{|c|c|c|c|c|c|}
\hline \multirow[t]{2}{*}{ Parameter } & \multicolumn{4}{|c|}{ No. of points of stage in parameter } & \multirow{2}{*}{$\begin{array}{l}\text { No. of } \\
\text { maximal } \\
\text { possible } \\
\text { points of } \\
\text { parameter }\end{array}$} \\
\hline & $I$ & II & III & IV & \\
\hline A & 14 & $2 !$ & $33_{4}^{3}$ & 5 & 5 \\
\hline B & $1 \frac{2}{3}$ & $3 !$ & 5 & - & 5 \\
\hline $\mathrm{C}$ & 11 & $2 \frac{1}{2}$ & $3 i$ & 5 & 5 \\
\hline D & 10 & 20 & 30 & 40 & 40 \\
\hline E & $13 !$ & $26_{3}^{2}$ & 40 & - & 40 \\
\hline $\mathbf{F}$ & $1:$ & $3 !$ & 5 & -- & 5 \\
\hline \multicolumn{5}{|c|}{$A \cdot B \cdot C-D \cdots E+F$} & 100 \\
\hline
\end{tabular}

The points of each parameter were added up by the clinician to a point score for NVD overall grading:

Table 2 Overall grading score of NVD

\begin{tabular}{lc}
\hline NVD overall grade & No. of points \\
\hline 1 & $1-30$ \\
2 & $31-60$ \\
3 & $61-90$ \\
4 & $91-100$ \\
\hline
\end{tabular}

The higher the number of points the more advanced is the disc neovascularisation.

According to this scheme four standard photographs (Figs. 5a-d) were established to facilitate NVD grading either by the point system or by comparing any disc photograph to the overall grade standard photograph it most closely resembles. 

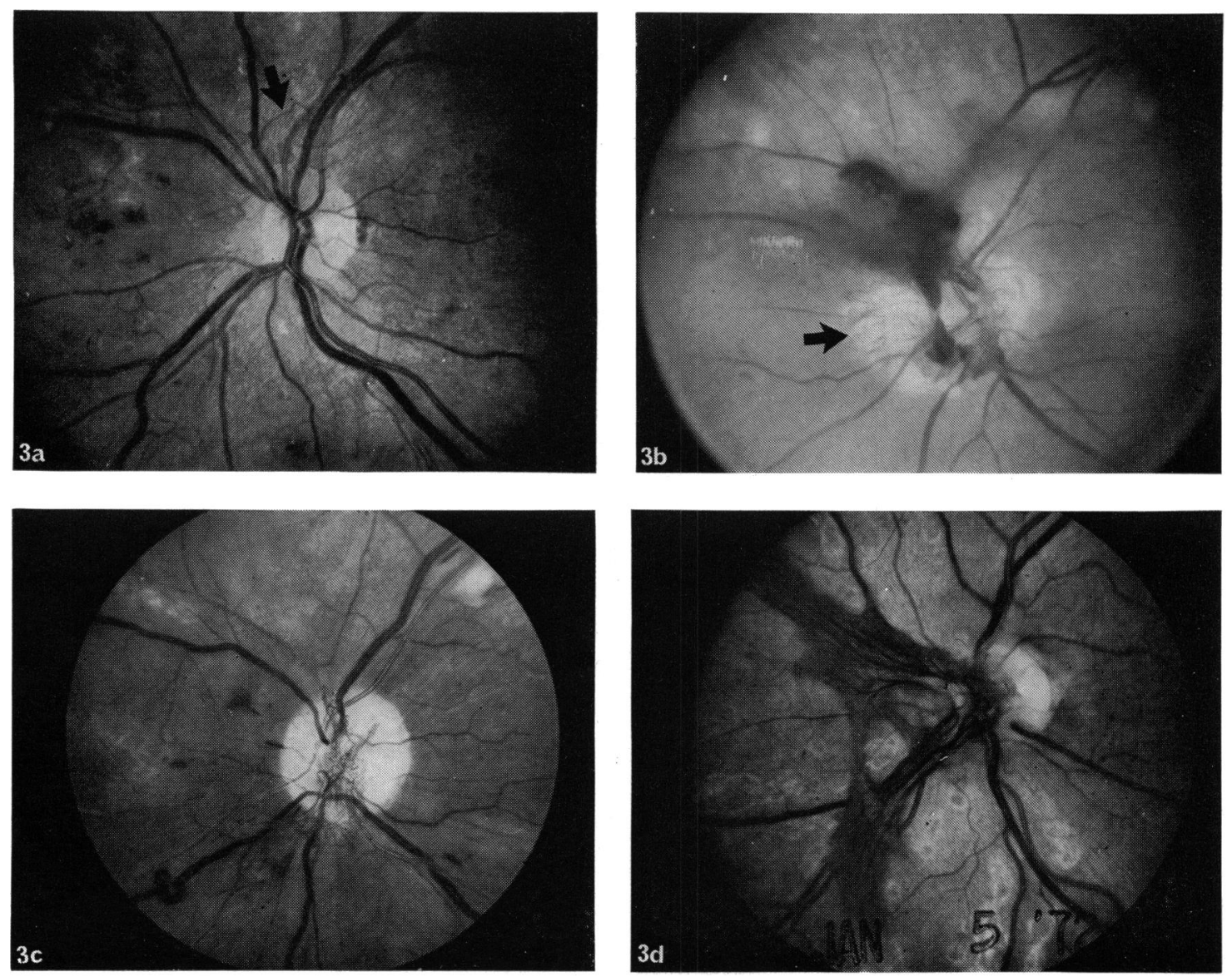

Figs. 3a-d The average density of disc neovascularisation is graded in 4 stages. Examples of each stage are demonstrated: 3a. Density of vessels stage I. 3b. Density of vessels stage II. 3c. Density of vessels stage III. 3d. Density of vessels stage IV.

Grading classification for the fibrous proliferation at the disc (FPD) was separately determined, and was based on 3 parameters:

A. The area of proliferation, with I-IV stages, as described for NVD area of proliferation.

B. Plane of proliferation, with I-III stages, as described for NVD plane of proliferation.

C. The density of the fibrous tissue (stages I-III), determined according to $6 a, 6 b$, and $6 d$ standard photographs. Here again, according to our observations on the natural history of FPD since 1960, each parameter received a relative score: parameter $\mathrm{A}$ received a score of 40 points toward 100 , and parameters $\mathrm{B}$ and $\mathrm{C}$ received 30 points each. Each stage within each parameter received the points as follows: 

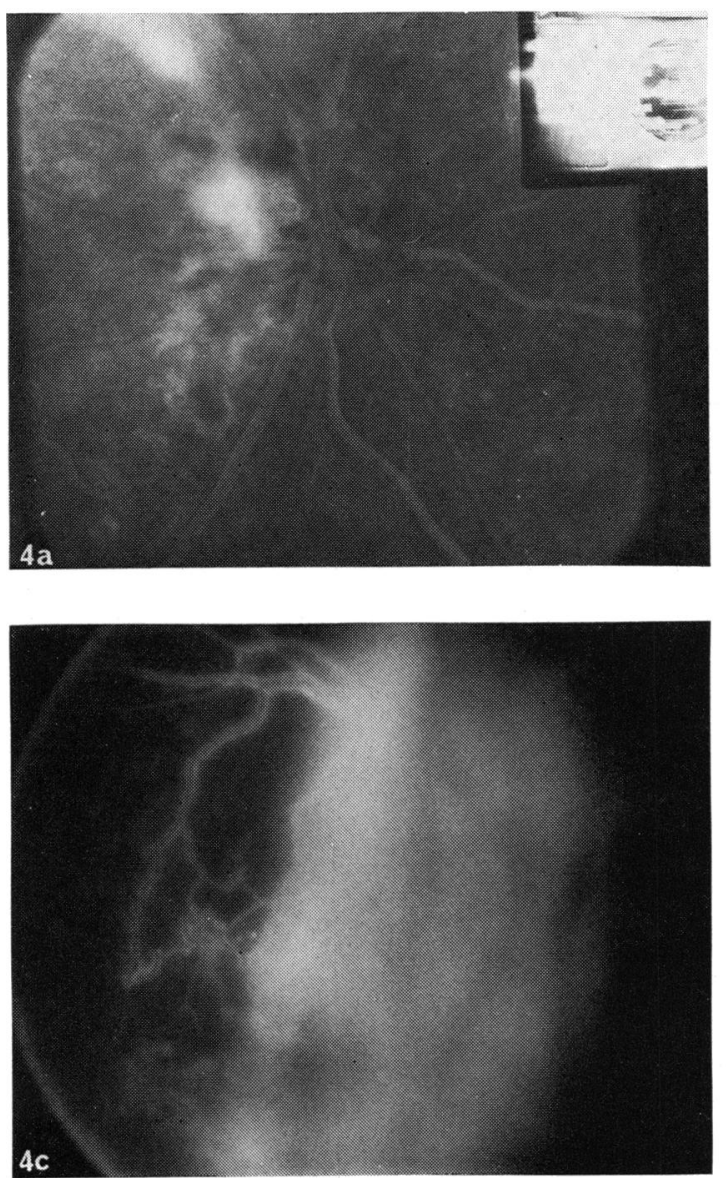

The points of each parameter were added up by the clinician to a point score for FPD overall grading:

Table 4 Overall grading score of FPD

\begin{tabular}{ll}
\hline FPD overall grade & No. of points \\
\hline 1 & $1-30$ \\
2 & $31-60$ \\
3 & $61-90$ \\
4 & $91-100$ \\
\hline
\end{tabular}

The higher the number of points, the more advanced is the disc fibrous proliferation.

According to this, the following 4 standard photographs for an overall FPD grading were established, so that any examined disc fibrous proliferation may be graded either by the point

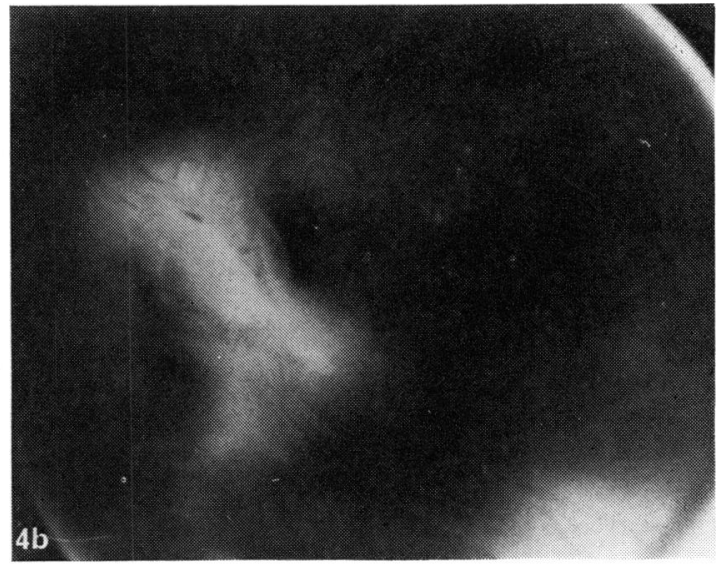

Figs. 4a-c Fluorescein leakage is graded from stage I, indicating a mild dye leakage, up to stage III, with maximum dye leakage evaluated in the films at approximately 1-2 minutes following the appearance of the dye in the retinal circulation: 4a. Stage I fluorescein leakage from the disc and from vessels inferior to disc showing mild amount of leaking dye. 4b. Stage II fluorescein leakage showing moderate amount of dye in the vitreous. 4c. Stage III fluorescein leakage showing heavy dye leakage into the vitreal space.

system or by comparing it to the overall grade standard photograph it most closely resembles (Fig. 6).

\section{Discussion}

Patients with neovascularisation emanating from the disc have a poor visual prognosis, and there is a high incidence of vitreous haemorrhage as well as fibrous proliferation and traction retinal detachment.

Any grading system for proliferation at the optic disc is arbitrary and based upon various considerations embedded in the author's clinical judgment as being relatively more significant. Most morphological descriptions of optic nerve head proliferation have been based on the general appearance of the disc, with the observer noting whether it was 'worse' or 'better' with time. A more systematic classification was laid by Kohner et al., ${ }^{3}$ and it incorporated morphological as well as some quantitative para- 

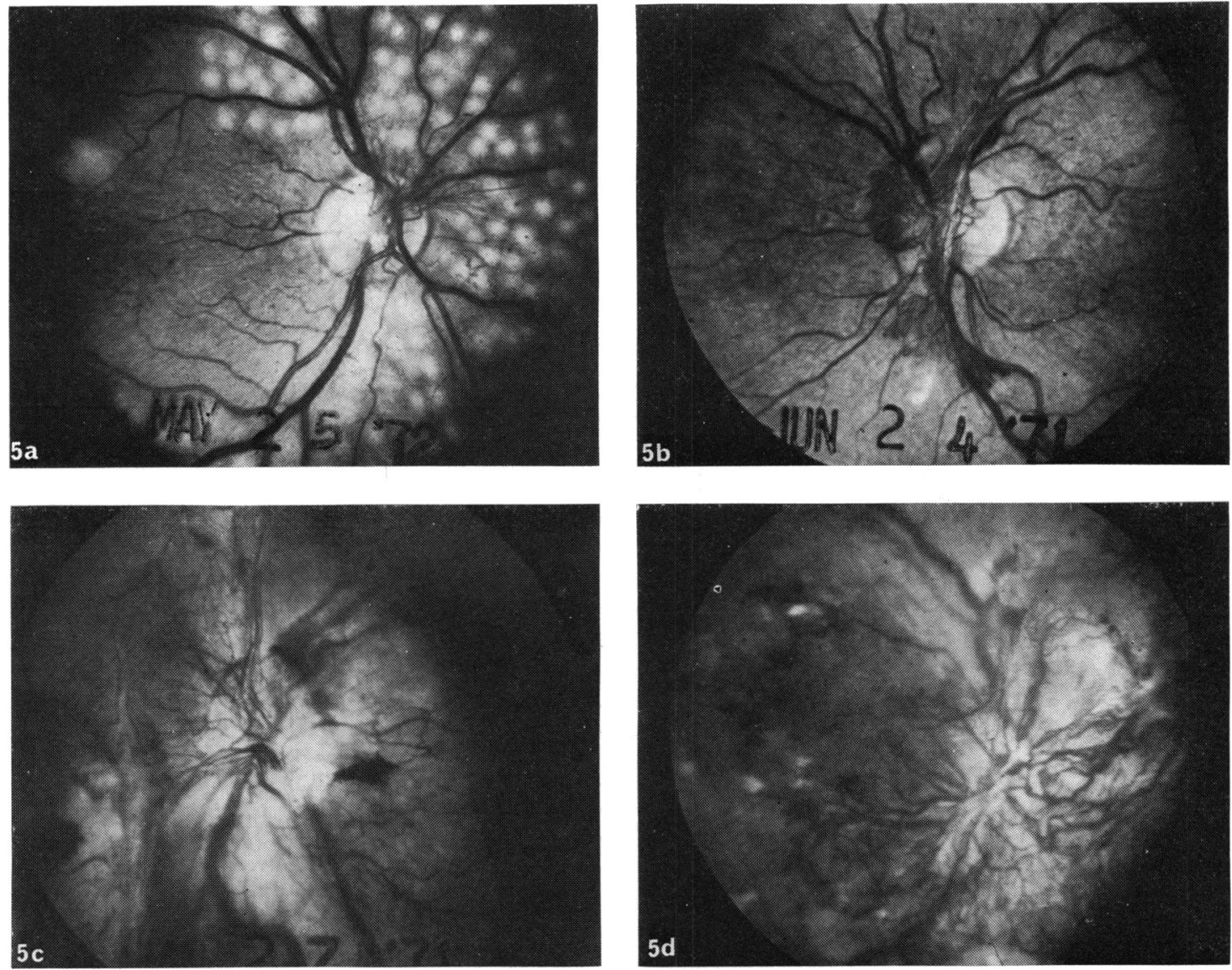

Figs. 5a-d Overall grading of disc neovascularisation: 5a. Grade 1 neovascularisation of the disc. 5b. Grade 2 neovascularisation of the disc. 5c. Grade 3 neovascularisation of the disc. 5d. Grade 4 neovascularisation of the disc.

meters. It has later been used by the British Multicentre Photocoagulation Trial Group. ${ }^{8}$ However, this system also had limitations, some of them expressed by the authors themselves: (1) Perceptible alterations in neovascularisation occur, without change in grade. (2) Density of new vessels is not graded. (3) A distinction between NVD and FPD was not used in that classification. Instead, when fibrous proliferation occurred, the NVD grading by that system was 'frozen'; NVD grading remained, therefore, almost unchanged, and the significance of moving from primarily neovascular proliferation to primarily fibrous proliferation could not be assessed. (4) The total area of NVD and FPD and the plane of proliferation could not be incorporated in the grading system, since stereophotography was originally not used. (5) Calibre of the new blood vessels was not graded.
L'Esperance's classification, ${ }^{4}$ although detailed in some morphological aspects, did not establish a taxonomy of grading and of evaluating progression or regression of either form of disc proliferation. The same is true of Hercules et al.'s classification, ${ }^{5}$ which is actually a modification of l'Esperance's classification. The Diabetic Retinopathy Study Research Group classification ${ }^{6} 7$ did not go into all details of NVD and FPD components.

The present study attempts to overcome these disadvantages. It is based on detailed parameters according to the experience accumulated out of approximately 600 patients with NVD and FPD, who have been observed since a natural history study was started in 1960 . This classification also can discriminate between the evolution of NVD and FPD. New vessels are more apt to bleed, while fibrous tissue is more apt to cause gliosis of the 

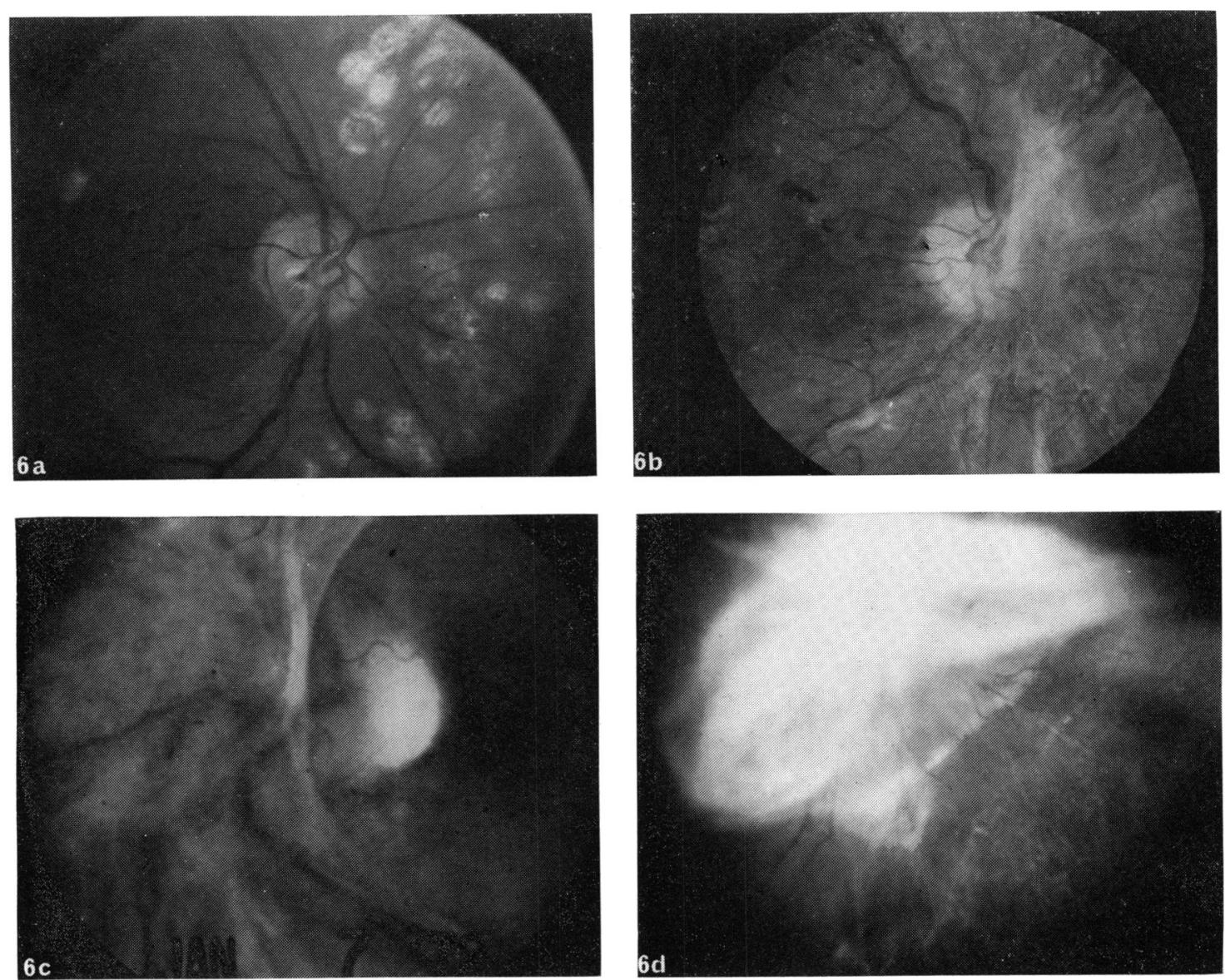

Figs. 6a-d Gradation of the amount of fibrous proliferation present at the disc: 6a. Grade 1 fibrous proliferation at the disc (also stands for density stage I of FDP). 6b. Grade 2 fibrous proliferation at the disc (also stands for density stage II of FPD). 6c. Grade 3 fibrous proliferation at the disc. 6d. Grade 4 fibrous proliferation at the disc (also stands for density stage III of FPD).

retina and traction retinal detachment. We often see that disc new vessels are replaced by fibrotic tissue, and the present taxonomy can follow the reciprocity between these 2 forms of disc proliferation.

By this taxonomy the variations in a particular disc can be followed for a time period without using general terms such as 'progression' or 'improvement', by recording the scores for the examined parameters. This taxonomy may permit a better understanding of follow-up of the natural history and results of treatment of disc proliferation in terms that can be used at different clinical centres by different examiners, and may serve until another classification is introduced according to future new knowledge.
Visual acuity was not incorporated in establishing this grading system. The visual acuity is undoubtedly the criterion by which the patient judges the changes of this disease and response to treatment. Nevertheless, there are so many other factors involved in determining visual acuity, such as macular oedema and degree of vitreous haemorrhage-and quite often vision at a certain time can be good in the presence of severe disc pathology and vice versathat using visual acuity in the grading system would create an additional unnecessary bias in interpreting the status of the optic nerve head.

\section{References}

${ }^{1}$ Davis MD, Norton EWD, Myers FL. Airlie classification of diabetic retinopathy. In: Goldberg MF, Fine SL (eds), 
Symposium on the Treatment of Diabetic Retinopathy Washington: US Public Health Service Publication No. 1890,$1968 ; 7$.

${ }^{2}$ Lee P F, McMeel TW, Schepens CL, Field RA. A new classification of diabetic retinopathy. Am J Ophthalmol 1966; 62:207-219.

${ }^{3}$ Kohner EM, Panisset A, Cheng H, Fraser TR. Diabetic retinopathy: new vessels arising from the optic disc: I. Grading system and natural history. Diabetes 1971;20:816823.

${ }^{4}$ L'Esperance FA. Argon laser photocoagulation in diabetic lesions. In: Lynn JR, Snyder WB, Vaiser A eds. Diabetic Retinopathy. New York: Grune and Stratton, 1974.

${ }^{5}$ Hercules BL, Gayed II, Lucas SB, Jeacock J. Peripheral retinal ablation in the treatment of proliferative diabetic retinopathy: a three-year interim report of a randomised controlled study using the argon laser. $\mathrm{Br} J$ Ophthalmol $1977 ; 61: 555-563$.

${ }^{6}$ Diabetic Retinopathy Study Research Group. Preliminary report on effects of photocoagulation therapy. $A m J$ Ophthalmol 1976;81:383-396.

'Diabetic Retinopathy Study Research Group. Photocoagulation treatment of proliferative diabetic retinopathy: the second report of diabetic retinopathy study findings. Am Acad Ophthalmol 1978; 85:82-106.

${ }^{8}$ British Multicentre Photocoagulation Trial. Proliferative diabetic retinopathy: treatment with xenon arc photocoagulation. Br Med J 1977; 1:739-741. 